PROCEEDINGS OF THE

AMERICAN MATHEMATICAL SOCIETY

Volume 130, Number 5, Pages 1377-1381

S 0002-9939(01)06389-4

Article electronically published on December 20, 2001

\title{
CRITICAL POINTS OF THE AREA FUNCTIONAL OF A COMPLEX CLOSED CURVE ON THE MANIFOLD OF KÄHLER METRICS
}

\author{
ABEL CASTORENA
}

(Communicated by Mohan Ramachandran)

\begin{abstract}
We consider a compact complex manifold $M$ of dimension $n$ that admits Kähler metrics and we assume that $C \hookrightarrow M$ is a closed complex curve. We denote by $\mathcal{K C}(1)$ the space of classes of Kähler forms $[\omega] \in H^{1,1}(M, \mathbb{R})$ that define Kähler metrics of volume 1 on $M$ and define $\mathbf{A}_{C}: \mathcal{K C}(1) \rightarrow \mathbb{R}$ by $\mathbf{A}_{C}([\omega])=\int_{C} \omega=$ area of $C$ in the induced metric by $\omega$. We show how the Riemann-Hodge bilinear relations imply that any critical point of $\mathbf{A}_{C}$ is the strict global minimum and we give conditions under which there is such a critical point $[\omega]$ : A positive multiple of $[\omega]^{n-1} \in H^{2 n-2}(M, \mathbb{R})$ is the Poincaré dual of the homology class of $C$. Applying this to the Abel-Jacobi map of a curve into its Jacobian, $C \hookrightarrow J(C)$, we obtain that the Theta metric minimizes the area of $C$ within all Kähler metrics of volume 1 on $J(C)$.
\end{abstract}

\section{INTRODUCTION}

Let $M$ be a compact complex manifold of dimension $n$ that admit Kähler metrics. The Kähler cone

$$
\mathcal{K C}:=\left\{[\omega] \in H^{1,1}(M, \mathbb{R}) \mid \omega \text { is a Kähler form }\right\}
$$

is an open convex cone in the vector space $H^{1,1}(M, \mathbb{R})$. On $\mathcal{K C}$ we define the function

$$
\mathbf{V}: \mathcal{K C} \rightarrow \mathbb{R}^{+}, \quad \mathbf{V}([\omega])=\frac{1}{n !} \int_{M} \omega^{n}=\operatorname{Vol}(M, \omega)
$$

where $\omega$ is a Kähler form representing the class $[\omega]$ and $\operatorname{Vol}(M, \omega)$ denotes the volume of $M$ in the metric induced by $\omega$. Suppose that $C \hookrightarrow M$ is a closed complex curve in $M$. For $[\omega] \in \mathcal{K C}$, the area of $C$ in the induced metric of $\omega$ is given by

$$
\widetilde{\mathbf{A}}_{C}(\omega):=\int_{C} \omega=\text { area of } C .
$$

Received by the editors November 2, 2000.

2000 Mathematics Subject Classification. Primary 32Q15.

Key words and phrases. Kähler form, Kähler manifold, Riemann-Hodge bilinear relations, Jacobian of a curve. 
The function $\widetilde{\mathbf{A}}_{C}$ defines a functional in $H^{1,1}(M, \mathbb{R})$. In this paper we study the critical points of

$$
\mathbf{A}_{C}:=\left.\widetilde{\mathbf{A}}_{C}\right|_{\mathcal{K C}(1)}: \mathcal{K C}(1) \rightarrow \mathbb{R}
$$

where $\mathcal{K C}(1):=\mathbf{V}^{-1}(1)$ is the hypersurface of cohomology classes of Kähler forms that define Kähler metrics of volume 1 on $M$. We use the Riemann-Hodge bilinear relations to show that the set $\mathcal{D}_{1}:=\{[\omega] \in \mathcal{K C} \mid \mathbf{V}([\omega]) \geq 1\}$ is a strictly convex set where $\mathcal{K C}(1)$ is the boundary of $\mathcal{D}_{1}$. Let PD $[C]$ be the Poincare dual of the homology class of $C$ in $H^{2 n-2}(M, \mathbb{R})$.

Theorem. Let $M$ be a compact complex manifold of dimension $n$ that admit Kähler metrics and $C \hookrightarrow M$ be a complex closed curve in $M$. Then:

(i) any critical point of $\mathbf{A}_{C}$ is a strict global minimum, and

(ii) this critical point exists, call it $[\omega]$, if and only if

$$
P D([C])=\alpha[\omega]^{n-1} \text { for some } \alpha>0 .
$$

Let $C$ be a compact Riemann surface of genus $g \geq 2, J(C)$ the Jacobian of $C$ and $C \hookrightarrow J(C)$ the Abel-Jacobi map ([1, p. 235). In this case we have from Poincaré's formula ([1], p. 350) that $\mathrm{PD}[C]=\frac{\left[\omega_{0}\right]^{g-1}}{(g-1) !}$, where $\omega_{0}$ is the 2 -form on $J(C)$ invariant under translations representing the first Chern class of the line bundle defined by the Riemann Theta divisor of $J(C)$. In this case $\omega_{0}$ defines a canonical flat Kähler metric on $J(C)$, called the Theta metric.

Corollary. The Theta metric minimizes the area of the Abel-Jacobi curve C within all Kähler metrics of volume 1 on the Jacobian $J(C)$.

\section{The Hessian of $\mathbf{V}$ and the Riemann-Hodge bilinear Relations}

Lemma 2.1. Let $[\omega] \in \mathcal{K C}$. Then for $[\eta] \in T_{[\omega]} \mathcal{K C}=H^{1,1}(M, \mathbb{R})$ a tangent vector, we have

$$
d \mathbf{V}_{[\omega]}([\eta])=\int_{M} *_{\omega} \omega \wedge \eta=:\langle\omega, \eta\rangle_{\omega}
$$

where $\langle,\rangle_{\omega}$ denotes the inner product on $H^{1,1}(M, \mathbb{R})$ and $*_{\omega}$ is the star operator induced by $\omega$.

Proof. This is a simple computation:

$$
\begin{aligned}
d \mathbf{V}_{\omega}(\eta) & =\lim _{t \rightarrow 0} \frac{1}{t}(\mathbf{V}(\omega+t \eta)-\mathbf{V}(\omega))=\frac{1}{n !} \lim _{t \rightarrow 0} \frac{1}{t}\left(\int_{M}(\omega+t \eta)^{n}-\int_{M} \omega^{n}\right) \\
& =\frac{1}{n !} \lim _{t \rightarrow 0} \frac{1}{t}\left(n t \int_{M}\left(\omega^{n-1} \wedge \eta\right)+\left(\left(\begin{array}{l}
n \\
2
\end{array}\right) \cdot t^{2} \int_{M}\left(\eta^{2} \wedge \omega^{n-2}\right)\right)+\cdots\right) \\
& =\frac{n}{n !} \int_{M} \omega^{n-1} \wedge \eta=\frac{1}{(n-1) !} \int_{M} \omega^{n-1} \wedge \eta
\end{aligned}
$$

Since $*_{\omega} \omega=\frac{\omega^{n-1}}{(n-1) !}$, we obtain that $d \mathbf{V}_{\omega}(\eta)=\langle\omega, \eta\rangle_{\omega}$.

Remark. Lemma 2.1 implies that Kernel $d \mathbf{V}_{\omega}=H_{0}^{1,1}([\omega])$, where $H_{0}^{1,1}([\omega])$ is the space of primitive cohomology classes of $\omega([1]$, p. 122). This lemma also implies 
that $\mathcal{K C}(1):=\mathbf{V}^{-1}(1)$ is a smooth hypersurface and for $[\omega] \in \mathcal{K C}(1), T_{[\omega]} \mathcal{K C}(1)=$ $H_{0}^{1,1}([\omega])$.

For $[\omega] \in \mathcal{K C}$, we have the bilinear form $Q_{\omega}: H^{1,1}(M, \mathbb{R}) \times H^{1,1}(M, \mathbb{R}) \rightarrow \mathbb{R}$ defined by

$$
Q_{\omega}\left(\eta_{1}, \eta_{2}\right)=\int_{M} \eta_{1} \wedge \eta_{2} \wedge \omega^{n-2} .
$$

Riemann-Hodge bilinear relations for $H^{1,1}(M, \mathbb{R})$. The form $Q_{\omega}$ is negative definite on primitive cohomology $H_{0}^{1,1}([\omega])([1]$, p. 123).

Lemma 2.2. Let $[\omega] \in \mathcal{K C}$ and $[\eta],[\zeta] \in T_{[\omega]} \mathcal{K C}=H^{1,1}(M, \mathbb{R})$. Then we have

$$
\text { Hess } \mathbf{V}_{[\omega]}([\eta],[\zeta])=\frac{1}{(n-2) !} \int_{M} \omega^{n-2} \wedge \eta \wedge \zeta
$$

and Hess $\left.\mathbf{V}_{\omega}\right|_{H_{0}^{1,1}([\omega])}$ is negative definite.

Proof. By definition, Hess $\mathbf{V}_{[\omega]}: H^{1,1}(M, \mathbb{R}) \times H^{1,1}(M, \mathbb{R}) \rightarrow \mathbb{R}$ is

$$
\begin{aligned}
& \operatorname{Hess}_{[\omega]}([\eta],[\zeta])=\lim _{t \rightarrow 0} \frac{1}{t}\left(d \mathbf{V}_{\omega+t \eta}(\zeta)-d \mathbf{V}_{\omega}(\zeta)\right) \\
& =\frac{1}{(n-1) !} \lim _{t \rightarrow 0}\left(\left(\int_{M}(\omega+t \eta)^{n-1} \wedge \zeta\right)-\left(\int_{M} \omega^{n-1} \wedge \zeta\right)\right) \\
& =\frac{(n-1)}{(n-1) !} \int_{M} \omega^{n-2} \wedge \eta \wedge \zeta=\frac{1}{(n-2) !} \int_{M} \omega^{n-2} \wedge \eta \wedge \zeta .
\end{aligned}
$$

So the lemma follows from the Riemann-Hodge bilinear relations.

\section{The Set $\mathcal{D}_{1}$ IS StRictly CONVEX}

Let $\mathcal{B} \subset \mathbb{R}^{n}$ be a set with nonempty interior: int $\mathcal{B} \neq \emptyset$. $\mathcal{B}$ is strictly convex if for all $x, y \in \mathcal{B}, x \neq y$, we have that $t x+(1-t) y \in \operatorname{int} \mathcal{B}$ for all $t \in(0,1)$.

Lemma 3.1. Let $\mathcal{B} \subset \mathbb{R}^{n}$ be a strictly convex set with smooth boundary $\partial \mathcal{B}$. Let $\widetilde{T}: \mathbb{R}^{n} \rightarrow \mathbb{R}$ be a linear functional. Consider the restriction $T:=\left.\widetilde{T}\right|_{\mathcal{B}}: \mathcal{B} \rightarrow \mathbb{R}$. If $p \in \mathcal{B}$ is a strict local minimum of $T$, then $p$ is the strict global minimum of $T$.

Proof. This follows since $\mathcal{B}-\{p\}$ is contained in one of the half spaces obtained as the complement of the tangent hyperplane to $p \in \partial \mathcal{B}$.

Proposition 3.2. Let $\mathcal{B} \subset \mathbb{R}^{n}$ be open and convex. Let $f: \mathcal{B} \rightarrow \mathbb{R}$ be a function of class $C^{2}$ such that it satisfies the following conditions:

(a) for each $x \in \mathcal{B}, d f(x) \neq 0$,

(b) $\left.(\text { Hess } f)_{x}\right|_{\text {Kernel } d f(x)}$ is negative definite for all $x \in \mathcal{B}$.

Then the set $\mathcal{B}_{1}:=\{x \in \mathcal{B} \mid f(x) \geq 1\}$ is strictly convex.

Proof. Let $x, y \in \mathcal{B}_{1}$ be $x \neq y$, and consider the line $r(t)=t x+(1-t) y, t \in[0,1]$. Let $F:[0,1] \rightarrow \mathbb{R}$ be defined by $F(t)=f(r(t))$. We have the following cases:

(i) If $F^{\prime}(t)>0$ in $(0,1]$, then for all $t \in(0,1], F(t)>F(0) \geq 1$, hence $r((0,1]) \in$ int $\mathcal{B}_{1}$.

(ii) If $F^{\prime}(t)<0$ in $[0,1)$, then for all $t \in[0,1), F(t)>F(1) \geq 1$, hence $r([0,1)) \in$ int $\mathcal{B}_{1}$. 
(iii) If there exists a $t_{0} \in[0,1]$ such that $F^{\prime}\left(t_{0}\right)=0$, then $x-y \in \operatorname{Kernel} d f\left(r\left(t_{0}\right)\right)$. By hypothesis we have

$$
F^{\prime \prime}\left(t_{0}\right)=D^{2} f\left(r\left(t_{0}\right)\right)(x-y, x-y)<0,
$$

that is, $t_{0}$ is a local maximum. Hence, at any critical point $F$ has a local maximum, which clearly implies that $t_{0}$ is the unique global maximum of $F$. This implies that for all $t \in\left[0, t_{0}\right), F^{\prime}(t)>0$, that is, $F$ is increasing in $\left[0, t_{0}\right)$; and for all $t \in\left(t_{0}, 1\right]$, $F^{\prime}(t)<0$, then $F$ is decreasing in $\left(t_{0}, 1\right]$. This implies that $F(t)>1$ for all $t \in(0,1)$, in other words, $r((0,1)) \in$ int $\mathcal{B}_{1}$.

Then (i), (ii), (iii) prove that $\mathcal{B}_{1}$ is strictly convex.

Corollary 3.3. The set $\mathcal{D}_{1}:=\{[\omega] \in \mathcal{K C} \mid \mathbf{V}([\omega]) \geq 1\}$ is strictly convex.

Proof. By Lemmas 2.1 and 2.2 we have that the function $\mathbf{V}$ satisfies the hypothesis of Proposition 3.2.

\section{Minimizing $\mathbf{A}_{C}$ ON $\mathcal{D}_{1}$}

Lemma 4.1. If $[\omega] \in \mathcal{K C}(1)$ is a critical point of $\mathbf{A}_{C}$, then $[\omega]$ is the global minimum of $\widetilde{\mathbf{A}}_{C}$ on $\mathcal{D}_{1}$. In particular $[\omega]$ is the global minimum of $\mathbf{A}_{C}$ on $\mathcal{K C}(1)$.

Proof. Suppose that $[\omega] \in \mathcal{K C}(1)$ is a critical point of $\mathbf{A}_{C}$. This implies that there exists a $\lambda_{0} \in \mathbb{R}$ such that $\left(\lambda_{0},[\omega]\right)$ is a critical point of the Lagrange function

$$
L: \mathbb{R} \times \mathcal{K C} \rightarrow \mathbb{R}, \quad L(\lambda, \omega)=\widetilde{\mathbf{A}}_{C}(\omega)-\lambda \mathbf{V}(\omega) .
$$

It is easy to see that $\lambda_{0}>0$. We have that the Hessian of $\widetilde{\mathbf{A}}_{C}$ is zero since it is linear. From Lemma 2.2, Hess $\left.\mathbf{V}_{\omega}\right|_{H_{0}^{1,1}([\omega])}$ is negative definitive; then

$$
\text { Hess }\left.L\left(\lambda_{0},[\omega]\right)\right|_{H_{0}^{1,1}([\omega])}=-\lambda_{0} \text { Hess }\left.\mathbf{V}_{\omega}\right|_{H_{0}^{1,1}([\omega])}
$$

is positive definite. Using the second derivate test criteria we have that $[\omega]$ is a local strict minimum of $\mathbf{A}_{C}$ on $\mathcal{K C}(1)$. This implies that there exists an open neighbourhood $U$ of $[\omega]$ in $\mathcal{K C}(1)$ such that $\mathbf{A}_{C}([\omega])<\mathbf{A}_{C}([\tilde{\omega}])$ for all $[\tilde{\omega}] \in U-$ $\{[\omega]\}$. Note that $W=\{t[\tilde{\omega}] \mid t \geq 1,[\tilde{\omega}] \in U\}$ is an open set in $\mathcal{D}_{1}$. By linearity we have that $\mathbf{A}_{C}([\omega])<t \mathbf{A}_{C}([\tilde{\omega}])$ for all $t[\tilde{\omega}] \in W$. Then $[\omega]$ is a strict local minimum of $\widetilde{\mathbf{A}}_{C}$ on $\mathcal{D}_{1}$. Applying lemma 3.1 we have that $[\omega]$ is the global minimum of $\widetilde{\mathbf{A}}_{C}$ on $\mathcal{D}_{1}$. In particular $[\omega]$ is the global minimum of $\mathbf{A}_{C}$ on $\mathcal{K C}(1)$.

Proof of the Theorem. Lemma 4.1 proves part (i).

To prove part (ii) suppose that PD $[C]=\alpha[\omega]^{n-1},[\omega] \in \mathcal{K C}(1), \alpha>0$. Then it is easy to see that $d\left(\widetilde{\mathbf{A}}_{C}\right)_{[\omega]}-\lambda_{0} d \mathbf{V}_{[\omega]}=0, \lambda_{0}=\alpha(n-1)$ !. Then by Lagrange multipliers we have that $[\omega]$ is a critical point of $\mathbf{A}_{C}$.

Now suppose that $[\omega] \in \mathcal{K C}(1)$ is a critical point of $\mathbf{A}_{C}$, that is, $d \mathbf{V}_{[\omega]} \wedge$ $\left(d \widetilde{\mathbf{A}}_{C}\right)_{[\omega]}=0$. Then for $[\eta],[\zeta] \in T_{[\omega]} \mathcal{K C}$ we have

$$
d \mathbf{V}_{[\omega]}([\eta]) d\left(\widetilde{\mathbf{A}}_{C}\right)_{[\omega]}([\zeta])=d \mathbf{V}_{[\omega]}([\zeta]) d\left(\widetilde{\mathbf{A}}_{C}\right)_{[\omega]}([\eta]) .
$$

In particular we can take $[\zeta]=[\omega]$ and $[\eta] \in H_{0}^{1,1}([\omega])$, that is, $\langle\omega, \eta\rangle_{\omega}=0$. By Lemma 2.1 and (4.1) we have that $d\left(\widetilde{\mathbf{A}}_{C}\right)_{[\omega]}([\eta])=0$. By linearity of $\widetilde{\mathbf{A}}_{C}$, $d\left(\widetilde{\mathbf{A}}_{C}\right)_{[\omega]}([\eta])=\widetilde{\mathbf{A}}_{C}([\eta])$, then

$$
0=\int_{C} \eta=\int_{M} \eta \wedge *_{\omega}\left(*_{\omega}(\mathrm{PD}[C])\right)=\left\langle\eta, *_{\omega}(\mathrm{PD}[\mathrm{C}])\right\rangle_{\omega}
$$


for all $[\eta] \in H_{0}^{1,1}([\omega])$. Hence there exists $r_{1} \in \mathbb{R}$ such that $*_{\omega} \mathrm{PD}([C])=r_{1}[\omega]$. From the fact that $C$ is a complex curve there exists a positive real number $r_{2}$ such that $\mathrm{PD}[C]=r_{2} \frac{[\omega]^{n-1}}{(n-1) !}$, we take $\alpha=\frac{r_{2}}{(n-1) !}$.

Proof of the Corollary. By Poincaré's formula we have that

$$
\operatorname{PD}[C]=\frac{\left[\omega_{0}\right]^{n-1}}{(n-1) !}
$$

Applying the Theorem we obtain the Corollary.

Example 1. We consider the projective line $\mathbb{C P}^{1}$ and fix $p \in \mathbb{C P}^{1}$. Let $M=$ $\mathbb{C P}^{1} \times \mathbb{C P}^{1}$ and define

$$
\jmath: \mathbb{C P}^{1} \hookrightarrow M, \quad \jmath(x)=(x, p) .
$$

$C:=\jmath\left(\mathbb{C P}^{1}\right)$. We have projections $\pi_{1}, \pi_{2}: M \rightarrow \mathbb{C P}^{1}, \pi_{1}(x, y)=x, \pi_{2}(x, y)=y$, and let $\omega$ be the Fubini-Study form of $\mathbb{C P}^{1} . \omega_{t}:=t \pi_{1}^{*}(\omega)+\frac{1}{t} \pi_{2}^{*}(\omega), t>0$, are Kähler forms on $M$ of volume 1. Clearly $\int_{C} \omega_{t}=t$. In this case we have that infimum $\left\{\mathbf{A}_{C}\left(\omega_{t}\right)\right\}=0$. Hence $\mathbf{A}_{C}$ has no minimum on $\mathcal{K C}(1)$.

However when we consider the diagonal map $\triangle: \mathbb{C P}^{1} \hookrightarrow M, \triangle(x)=(x, x)$, and $C:=\triangle\left(\mathbb{C P}^{1}\right)$, we have $\mathrm{PD}[C]=\pi_{1}^{*}(\omega)+\pi_{2}^{*}(\omega)$, and by the Theorem the minimum of $\mathbf{A}_{C}$ is 1 and is obtained in $\frac{1}{2}\left[\pi_{1}^{*}(\omega)+\pi_{2}^{*}(\omega)\right]$. This example can be generalized to $\mathbb{C P}^{1} \hookrightarrow \underbrace{\mathbb{C P}^{1} \times \cdots \times \mathbb{C P}^{1}}_{k \text {-times }}$.

Example 2. Let $L \rightarrow M$ be an ample line bundle on a complex $n$-dimensional manifold $M$ and $c_{1}(L)=[\omega]$ be the first Chern class of $L$. Then there exist complex closed curves $C \hookrightarrow M$ such that $\mathrm{PD}[C]=\alpha[\omega]^{n-1}, \alpha>0$ : Embed $M \hookrightarrow \mathbb{C P}^{N}$ with a multiple of $L$, then intersect $M$ with generic hyperplanes of $\mathbb{C P}^{N}$ untill one obtains a curve $C$ as desired.

\section{REFERENCES}

[1] P. Griffiths, J. Harris, Principles of Algebraic Geometry, John Wiley and Sons, 1994. MR 95d:14001

[2] A. Weil, Introduction á l'Étude des Variétés Kählériennes, Hermann and Cie, Paris, 1958. MR 22:1921

Cimat, AP. 402, CP. 36000 Guanajuato, Gto. Mexico

E-mail address: abel@cimat.mx 